\title{
Regular solutions of the Einstein-Yang-Mills equations
}

\author{
J. A. Smoller and A. G. Wasserman \\ University of Michigan, Department of Mathematics, Ann Arbor, Michigan 48109-1003
}

(Received 10 February 1995; accepted for publication 3 April 1995)

It is shown rigorously that any static symmetric solution of the Einstein-YangMills (YM) equations with SU(2) gauge group that is well behaved in the far field is one of three types: black hole, particlelike, or Riessner-Nordström-like (RN) solution. (In particular, any solution with finite ADM mass is well behaved in the far field.) Black-hole solutions are proven to be analytic at the event horizon and thus coincides with Bartnik-McKinnon (BM) black holes. Furthermore, the singularity in the metric at the event horizon can be transformed away by a Kruskal-like change of coordinates in which the YM field remains well behaved. Particlelike solutions are shown to satisfy the same initial conditions as the BM solutions at $r=0$. RN-like solutions will be considered elsewhere. (C) 1995 American Institute of Physics.

\section{INTRODUCTION}

The Einstein-Yang-Mills (EYM) equations with SU(2) gauge group have been discussed in many articles, see, e.g., Refs. 1-14; rigorous proofs for the existence of particlelike solutions, as well as black-hole solutions were first established in Refs. 7-9. In this article, we are mainly concerned with certain uniqueness results for these equations; in particular, we prove that any smooth black-hole solution which is defined in the "far field" (i.e., for $r \gg 1$ ) and has event horizon $\rho>0$, must be one of the solutions whose existence was first established in Ref. 9-there are no others. If $\rho=0$, i.e., particlelike solutions, the proof of uniqueness requires an additional differentiability assumption.

The EYM equations, with gauge group $\mathrm{SU}(2)$ can be written in the form ${ }^{2}$

$$
G_{i j}=\sigma T_{i j}, \quad d^{*} F_{i j}=0, \quad i, j=0, \ldots, 3 .
$$

Here $T_{i j}$ denotes the stress-energy tensor associated to the su(2)-valued Yang-Mills curvature two-form $F_{i j}, G_{i j}=R_{i j}-\frac{1}{2} R g_{i j}$ is the Einstein tensor computed with respect to the sought-for metric $g_{i j}, *$ denotes the Hodge star operator, and $\sigma$ is a universal constant. If we consider static, symmetric solutions, i.e., solutions depending only on $r$, and $G=\operatorname{SU}(2)$, then, ${ }^{1}$ we may write the metric as

$$
d s^{2}=-T(r)^{-2} d t^{2}+A(r)^{-1} d r^{2}+r^{2}\left(d \theta^{2}+\sin ^{2} \theta d \phi^{2}\right)
$$

and the Yang-Mills curvature two-form as

$$
F=w^{\prime} \tau_{1} d r \wedge d \theta+w^{\prime} \tau_{2} d r \wedge(\sin \theta d \phi)-\left(1-w^{2}\right) \tau_{3} d \theta \wedge(\sin \theta d \phi) .
$$

Here $(T, A)$, and $w=w(r)$ denote the unknown metric and connection coefficients, respectively, and $\tau_{1}, \tau_{2}, \tau_{3}$ form a (suitably normalized) basis for su(2). As discussed in Ref. 1, the EYM equations in this framework take the following form as a system of three ordinary differential equations:

$$
r A^{\prime}+\left(1+2 w^{\prime 2}\right) A=1-\frac{\left(1-w^{2}\right)^{2}}{r^{2}}
$$




$$
\begin{gathered}
r^{2} A w^{\prime \prime}+\left[r(1-A)-\frac{\left(1-w^{2}\right)^{2}}{r}\right] w^{\prime}+w\left(1-w^{2}\right)=0 \\
2 r A \frac{T^{\prime}}{T}=\frac{\left(1-w^{2}\right)^{2}}{r^{2}}+\left(1-2 w^{\prime 2}\right) A-1 .
\end{gathered}
$$

Since Eqs. (1.3) and (1.4) do not involve $T$, we can use these to solve for $A$ and $w$, and then solve Eq. (1.5) for $T$; thus we shall mainly concentrate our attention on Eqs. (1.3) and (1.4). We demand that our solutions be defined in the "far field," i.e.,

$$
1 \geqslant A(r)>0, \text { for sufficiently large } r .
$$

Solutions of Eqs. (1.3), (1.4) which satisfy Eq. (1.6) and

$$
A(r)<1 \text {, for } r>0
$$

will be called regular solutions; see Definition 2.1 below. [Such solutions have "positive mass," i.e., if the mass function $\mu(r)$ is defined by

$$
\mu(r)=r(1-A(r))
$$

then Eq. (1.7) is equivalent to $\mu(r)>0$.]

In this article we only consider solutions for which $1 \geqslant A(r) \geqslant 0$. [Note that if $A\left(r_{1}\right)=1$ and $r_{1}>0$, then $A(r)>1$ for $r<r_{1}, r$ near $r_{1}$. This holds because $A^{\prime}\left(r_{1}\right)<0$, as follows from Eq. (1.3), except for the trivial case of the flat Minkowski metric; for such $r, \mu(r)<0$.] It is a consequence of Theorem 2.1, proven below, that all regular solutions satisfy the following: there is an $M>0$ such that for all $r$ in the domain of definition

$$
\begin{gathered}
\mu(r) \leqslant M \quad \text { (finite total mass), } \\
w^{2}(r) \leqslant 1,
\end{gathered}
$$

and

$$
\lim _{r \rightarrow \infty}\left(A(r), w^{2}(r), w^{\prime}(r)\right)=(1,1,0) .
$$

Such solutions need not be defined for all $r$; we denote by $\rho$ the left-hand end point of the maximal interval such that $0<A(r) \leqslant 1$, and both $w$ and $w^{\prime}$ are finite. In this article we study these solutions in the region $\rho<r<\infty$, i.e., exterior to the "event horizon." In a future publication, we shall consider solutions in the interior of the event horizon. We also exclude here solutions for which $A(r)>1$ for some $r$. These "Reissner-Nordström"-like solutions are interesting, and will be studied in a forthcoming publication.

Observe that our nonlinear equations become singular when $A=0$ or $r=0$. It follows from standard results that solutions cease to exist only if the variables become unbounded at some finite $r$, or if the equations become singular. That is, it follows from standard results that our solution can be defined for all $r>0$ unless perhaps $\lim _{r \backslash \rho} A(r)=0$, for some $\rho$, or $w^{2}\left(r_{1}\right)>1$, for some $r_{1}>0$. Thus, we need only consider the cases

$$
\frac{\lim A}{r \backslash \rho}(r)=0, \text { for some } \rho>0
$$

or $\rho=0$ or $w^{2}>1$. If the equations become singular then the solution may or may not be defined at $r=\rho$. In Sec. II, Theorem 2.1, we show that $w^{2}>1$ cannot occur for solutions satisfying Eqs. (1.6) and (1.7). For solutions whose maximum domain of existence is $r>\rho$ (or $r \geqslant \rho$ ), where $\rho>0$, we 
prove that $\lim _{r \backslash \rho}\left(w(r), w^{\prime}(r)\right)$ lies on the curve $\mathscr{E}_{\rho}$ of "initial values" of black-hole solutions; cf. Eq. (3.3) below, and Ref. 9. In the Appendix, we show that such solutions are perforce, analytic at $r=\rho$, and hence coincide, by uniqueness, with the black-hole solutions whose existence was first obtained in Ref. 9. For solutions defined for all $r>0$, we show that such solutions can be extended to be $C^{1}$-functions for all $r \geqslant 0, w^{2}(0)=1, w^{\prime}(0)=0$, and $A(0)=1$. Because of the second-order singularity in Eq. (1.4) at $r=0$, we cannot show that these solutions agree with those in Refs. 6 and 7. It is an open question as to whether there exist particlelike solutions other than those described in Refs. 7 and 8.

In the last section, we prove that the singularity in the metric for EYM black holes, can be removed by a Kruskal-type ${ }^{15}$ change of variables, whereby the Yang-Mills field remains well behaved under this transformation.

\section{REGULAR SOLUTIONS SATISFY $w^{2} \leqslant 1$}

In this article, we study smooth solutions of Eqs. (1.3), (1.4); that is, solutions which for $r$ sufficiently large are both $C^{2}$-functions and satisfy $0<A(r) \leqslant 1$. For such solutions, we set

$$
\rho=\inf \{r: A(s) \geqslant 0 \text { for all } s>r\} \text {; }
$$

and (as remarked in Sec. I) we study these solutions on the range $\rho<r<\infty$.

Definition 2.1: A smooth solution of Eqs. (1.3), (1.4) is called regular if

$$
1 \geqslant A(r) \geqslant 0, \text { for } r>\rho \text {. }
$$

In this article we are concerned with classifying regular solutions. Note that the statement $A(r)>1$ means $\mu(r)<0$; cf. Eq. (1.7). Note too that if $(A, w)$ is a regular solution, then in fact, $A(r)<1$ for $r>\rho$, except in the case $\left(w^{2}(r), A(r)\right)=(1,1)$. For, if $A\left(r_{1}\right)=1$ for some $r_{1}>\rho$, then the solution can be continued to $r<r_{1}$, and (as we have remarked earlier) $A^{\prime}\left(r_{1}\right)<0$ so $A(r)>1$ for some $r$, $\rho<r<r_{1}$; the solution is thus not regular. Nonregular solutions, i.e., those with $A(r)>1$, will be considered in a forthcoming publication.

In this section we shall show that regular solutions must lie in the region $w^{2} \leqslant 1$. More precisely, we have the following theorem.

Theorem 2.1: Let $(A(r), w(r))$ be a regular solution of Eqs. (1.3), (1.4); then $w^{2}(r) \leqslant 1$, for all $r$ in the domain of definition.

This theorem will be a consequence of the following two results.

Proposition 2.2: If $(A, w)$ is a solution of Eqs. (1.3), (1.4) and if for some $r_{0}, A\left(r_{0}\right)>0$, $w^{2}\left(\mathrm{r}_{0}\right)>1$, and $\left(w w^{\prime}\right)\left(r_{0}\right)>0$, then there is an $r_{1}>r_{0}$ for which $A\left(r_{1}\right)=0$ and $w^{\prime}(r)$ is unbounded near $r_{1}$. Thus the solution cannot be continued beyond $r_{1}$, and hence is not regular.

Proposition 2.3: If $(A, w)$ is a solution of Eqs. (1.3), (1.4) and if for some $r_{0}, A\left(r_{0}\right)>0$, $w^{2}\left(r_{0}\right)>1$, and $\left(w w^{\prime}\right)\left(r_{0}\right)<0$, then there is an $r_{1}, 0<r_{1}<r_{0}$ such that $A(r)>0$ on the interval $\left[r_{1}, r_{0}\right]$, and $A\left(r_{1}\right)>1$, i.e., the solution is not regular at $r_{1}$.

Proof of Proposition 2.2: In proving this proposition, we only consider the case

$$
w\left(r_{0}\right)>1, \quad w^{\prime}\left(r_{0}\right)>0 ;
$$

the symmetric case $w\left(r_{0}\right)<-1, w^{\prime}\left(r_{0}\right)<0$ is treated similariy.

We assume that $(A, w)$ is a smooth solution of Eqs. (1.3), (1.4) and $A(r) \geqslant 0$ for $r>r_{0}$, and we shall show that this leads to a contradiction.

Our strategy for proving Proposition 2.2 is to regard the function $A(r)$ as a fixed positive function, defined for all $r>r_{0}$. We shall compare the solution $w(r)$ of Eq. (1.4) (with this fixed $A$ ) to the solution $\bar{w}$ of a simpler equation [see Eq. (2.6) below]. We shall show that $w^{\prime}(r)>\bar{w}^{\prime}(r)$ and that $\bar{w} \rightarrow \infty$ in finite $r$. For ease in comparison, we also consider an intermediate equation [Eq. (2.3) below]. 
Consider Eq. (1.4) which we write in the form

$$
r^{2} A w^{\prime \prime}+\Phi w^{\prime}+u w=0
$$

where

$$
\Phi(A, w, r)=r(1-A)-\frac{u^{2}}{r} \text { and } u=\left(1-w^{2}\right)
$$

Note that the region $w>0, w^{\prime}>0$ is invariant in forward $r$, since when $w^{\prime}=0$, Eq. (2.1) implies that $A w^{\prime \prime}>0$ and hence $w^{\prime \prime}>0$.

Now consider the equation

$$
r^{2} A \tilde{w}^{\prime \prime}+r \tilde{w}^{\prime}+\tilde{u} \tilde{w}=0, \quad \tilde{u}=\left(1-\tilde{w}^{2}\right)
$$

together with the "initial" conditions

$$
\tilde{w}\left(r_{0}\right)=w\left(r_{0}\right), \quad \tilde{w}^{\prime}\left(r_{0}\right)=w^{\prime}\left(r_{0}\right)
$$

[Here $A$ is the fixed function determined by Eqs. (1.3) and (1.4).] We now have the following result concerning the solutions of Eq. (2.1), and Eqs. (2.2), (2.4).

Lemma 2.4: $w^{\prime}(r)>\tilde{w}^{\prime}(r)$ for $r>r_{0}$ and hence $w(r)>\tilde{w}(r)$ for $r>r_{0}$.

Proof of Lemma 2.4: First observe that since $w\left(r_{0}\right)=\tilde{w}\left(r_{0}\right)$, it follows that at $r_{0}$

$$
r_{0}^{2} A\left(w^{\prime \prime}-\tilde{w}^{\prime \prime}\right)=-\left(\Phi-r_{0}\right) w^{\prime}>0
$$

since $\Phi\left(r_{0}\right)<r_{0}$. Hence as $A\left(r_{0}\right)>0$, it follows that

$$
w^{\prime \prime}\left(r_{0}\right)>\tilde{w}^{\prime \prime}\left(r_{0}\right)
$$

so that $w^{\prime}(r)>\tilde{w}^{\prime}(r)$ for $r>r_{0}, r$ near $r_{0}$. Now let $r_{1}$ (if it exists) be the first $r>r_{0}$ for which $w^{\prime}(r)=\tilde{w}^{\prime}(r)$. Since $-w\left(1-w^{2}\right)$ is a monotone increasing function of $w$ in the region $w^{2}>1$, and as noted above the region $w>1, w^{\prime}>0$ is invariant, we have

$$
-(u w)\left(r_{1}\right)>-(\tilde{u} \tilde{w})\left(r_{1}\right)
$$

Thus

$$
r_{1}^{2} A\left(r_{1}\right)\left(w^{\prime \prime}\left(r_{1}\right)-\tilde{w}^{\prime \prime}\left(r_{1}\right)\right)=-(u w)\left(r_{1}\right)+(\tilde{u} \tilde{w})\left(r_{1}\right)+w^{\prime}\left(r_{1}\right)\left(r_{1}-\Phi\left(r_{1}\right)\right)>0
$$

so $w^{\prime \prime}\left(r_{1}\right)>\tilde{w}^{\prime \prime}\left(r_{1}\right)$, and thus by the mean value theorem no such $r_{1}$ can exist. It follows that $w^{\prime}(r)>\tilde{w}^{\prime}(r)$ if $r>r_{0}$, and hence $w(r)>\tilde{w}(r)$, if $r>r_{0}$.

Now consider the equation

$$
r^{2} \bar{w}^{\prime \prime}+r \bar{w}^{\prime}+\bar{u} \bar{w}=0, \quad \bar{u}=1-\bar{w}^{2}
$$

together with "initial" conditions, which will be specified later. We shall compare solutions of Eqs. (2.3), (2.4) with solutions of Eq. (2.6). Before making this comparison, we need a lemma.

Lemma 2.5: Let $f(r)=r \tilde{w}^{\prime}(r)+\tilde{u}(r) \tilde{w}(r)$, where $\tilde{w}$ is the solution of Eqs. (2.3), (2.4). Then there is an $R>r_{0}$ such that $f(r)<0$ if $r \geqslant R$.

Proof: We have

$$
f^{\prime}=\left(2-3 \tilde{w}^{2}\right) \tilde{w}^{\prime}+r \tilde{w}^{\prime \prime}
$$

and when $f=0, r^{2} \tilde{w}^{\prime \prime} A=0$. Hence 


$$
\left.f^{\prime}\right|_{f=0}=\left(2-3 \tilde{w}^{2}\right) \tilde{w}^{\prime}<0
$$

and this shows that $f$ can cross zero at most once. Thus $f$ is either always positive for $r>r_{0}$, or $f$ is eventually negative. Suppose that $f(r)>0$ for all $r>r_{0}$; we shall show that this leads to a contradiction. Thus, if $f(r)>0$ for $r>r_{0}$, we have $r \tilde{w}^{\prime}+\tilde{u} \tilde{w}>0$ or (since $u w<0$ )

$$
\frac{d \tilde{w}}{-\tilde{u} \tilde{w}}>\frac{d r}{r}, \quad \text { or } \quad \frac{d \tilde{w}}{\tilde{w}^{3}-\tilde{w}}>\frac{d r}{r}, \quad r>r_{0} .
$$

Since

$$
\int_{\tilde{w}\left(r_{1}\right)}^{\infty} \frac{d w}{\tilde{w}^{3}-\tilde{w}}
$$

is bounded, and $\int_{r_{1}}^{\infty}(d r / r)=\infty$, we have a contradiction.

Now consider the solution of Eq. (2.6) together with the "initial" conditions

$$
\bar{w}(R)=\tilde{w}(R), \quad \bar{w}^{\prime}(R)=\tilde{w}^{\prime}(R),
$$

where $R$ is as described in Lemma 2.5. Recall too that $\tilde{w}(R)>1$, and $\tilde{w}^{\prime}(R)>0$. We now compare $\bar{w}$ with $\tilde{w}$.

Lemma 2.6: Suppose $\tilde{w}$ satisfies Eqs. (2.3), (2.4), and $\bar{w}$ satisfies Eqs. (2.6), (2.8). Then $\tilde{w}(r)>\bar{w}(r)$, and $\tilde{w}^{\prime}(r)>\bar{w}^{\prime}(r)$ if $r>R$.

Proof: We have

$$
\tilde{w}^{\prime \prime}(R)-\bar{w}^{\prime \prime}(R)=\frac{1}{R^{2} A(r)}(-f(R))-\frac{1}{R^{2}}(-f(R))=\frac{f(R)}{R^{2}}\left[1-\frac{1}{A(R)}\right]>0
$$

since $A(R)<1$. It follows that $\tilde{w}^{\prime}(r)>\bar{w}^{\prime}(r)$ if $r>R, r$ near $R$. Suppose now that there were a (smallest) $r_{1}>R$ for which $\tilde{w}^{\prime}\left(r_{1}\right)=\bar{w}^{\prime}\left(r_{1}\right)$; we shall show that no such point can exist. To do this, first note that $\tilde{w}\left(r_{1}\right)>\bar{w}\left(r_{1}\right)$, and hence $-\tilde{u}\left(r_{1}\right) \tilde{w}\left(r_{1}\right)>-\bar{u}\left(r_{1}\right) \bar{w}\left(r_{1}\right)$, since $\bar{w}\left(r_{1}\right)>1$. Thus

$$
r_{1}^{2} A\left(r_{1}\right) \tilde{w}^{\prime \prime}\left(r_{1}\right)-r_{1}^{2} \bar{w}^{\prime \prime}\left(r_{1}\right)=-\tilde{u}\left(r_{1}\right) \tilde{w}\left(r_{1}\right)+\bar{u}\left(r_{1}\right) \bar{w}\left(r_{1}\right)>0
$$

so

$$
\tilde{w}^{\prime \prime}\left(r_{1}\right)>A\left(r_{1}\right) \tilde{w}^{\prime \prime}\left(r_{1}\right)>\bar{w}^{\prime \prime}\left(r_{1}\right)
$$

and this is impossible by the mean-value theorem. Thus $\tilde{w}^{\prime}(r)>\bar{w}^{\prime}(r)$ if $r>R$, and hence $\tilde{w}(r)$ $>\bar{w}(r)$ if $r>R$.

Thus from Lemma 2.4, $w>\tilde{w}$, and from Lemma $2.6, \tilde{w}>\bar{w}$ for $r \geqslant R$; hence $w(r)>\bar{w}(r)$ for $r \geqslant R$; similarly $w^{\prime}(r)>\bar{w}^{\prime}(r)$ for $r \geqslant R$.

We shall next show that $\bar{w}^{\prime}$ becomes unbounded in finite $r$, namely, we have

Lemma 2.7: Let $\bar{w}$ solve Eqs. (2.6), (2.8); then there is an $\bar{r} \geqslant R$ for which

$$
\lim _{r \rightarrow \bar{r}} \bar{w}(r)=\infty, \quad \text { and } \quad \lim _{r \rightarrow \bar{r}} \bar{w}^{\prime}(r)=\infty
$$

Proof: In Eq. (2.6) make the change of independent variable $t=\ln r$, to get

$$
\ddot{w}+\bar{u} \bar{w}=0, \quad \cdot=d / d t .
$$


Then since $\bar{w}>1, \ddot{w}=-\bar{u} \bar{w}>0$, so $\dot{w}$ is an increasing function. If $T=\ln R$, and $t>T$, then this implies $\dot{w}(t)>\dot{w}(T)$, so that $\bar{w}(t) \rightarrow \infty$ as $t \rightarrow \infty$. Now Eq. (2.9) admits a Hamiltonian function, $H(r)=\left(\dot{w}^{2} / 2\right)+\left(\bar{w}^{2} / 2\right)-\left(\bar{w}^{4} / 4\right)$, and solutions of Eq. (2.9) are precisely the level curves of $H$. Thus $H_{0}=\left(\dot{w}^{2} / 2\right)+\left(\bar{w}^{2} / 2\right)-\left(\bar{w}^{4} / 4\right)$, so for large $t>T$

$$
\frac{\dot{w}^{2}(t)}{2}=H_{0}+\frac{\bar{w}^{4}(t)}{4}-\frac{\bar{w}^{2}(t)}{2}>\frac{2 \bar{w}^{4}(t)}{9}
$$

and hence $\dot{w}(t)>\frac{2}{3} \bar{w}^{2}(t)$. This implies that for some $\bar{t}>T, \lim _{t} \bar{t} \bar{w}(t)=\infty$, and hence $\lim _{r / \bar{r}} \bar{w}(r)=\infty$, where $\bar{r}=e^{\bar{t}}$. It then follows that $\lim _{r / \bar{r}} \bar{w}^{\prime}(r)=\infty$.

We can now complete the

Proof of Proposition 2.2: From Lemma 2.7, logether with the fact that $w^{\prime}(r)>\vec{w}^{\prime}(r)$ if $r \geqslant R$, we see that $w^{\prime}(r) \rightarrow \infty$ as $r \rightarrow \tilde{r}$, for some $\tilde{r} \leqslant \bar{r}$. This contradicts the fact that $w(r)$ is smooth for all $r \geqslant r_{0}$. It follows (since our solution is assumed to be smooth for all $r>r_{0}$ ) that $A\left(r_{1}\right)=0$ for some $r_{1}>r_{0}$, and as $A$ is continuous, we may assume $r_{1}$ is minimal with respect to this property. If $w^{\prime}$ is finite at $r_{1}$, then from Eq. (1.3) we see that

$$
r_{1} A^{\prime}\left(r_{1}\right)=1-\frac{\left(1-w^{2}\left(r_{1}\right)\right)^{2}}{r_{1}^{2}} .
$$

On the other hand from Eq. (1.4), we get

$$
\left[r_{1}-\frac{\left(1-w\left(r_{1}\right)^{2}\right)^{2}}{r_{1}}\right] w^{\prime}\left(r_{1}\right)+w\left(r_{1}\right)\left(1-w\left(r_{1}\right)^{2}\right)=0
$$

and this implies that $r_{1}>\left(1-w\left(r_{1}\right)^{2}\right)^{2} / r_{1}$. It follows from Eq. (2.9) that $A^{\prime}\left(r_{1}\right)>0$, and this is a contradiction. Thus $w^{\prime}$ must become unbounded at $r_{1}$.

We now consider the case where $w^{2}\left(r_{0}\right)>1$ and $\left(w w^{\prime}\right)\left(r_{0}\right)<0$, and give the

Proof of Proposition 2.3: Again we shall only consider the case $w\left(r_{0}\right)>1, w^{\prime}\left(r_{0}\right)<0$; the symmetric case $w\left(r_{0}\right)<-1, w^{\prime}\left(r_{0}\right)$ is treated similarly.

Now if $v=A w^{\prime}$, then (cf. Ref. 8) $v$ satisfies the equation

$$
v^{\prime}+\frac{2 w^{\prime 2} v}{r}+\frac{w\left(1-w^{2}\right)}{r^{2}}=0
$$

and hence $v^{\prime}>0$ in the region $w^{\prime}<0, w>0$. Since $v\left(r_{0}\right)<0, v(r)<v\left(r_{0}\right)$ if $r<r_{0}$. Thus for $r<r_{0}$, $w^{\prime}(r)<v\left(r_{0}\right) / A(r)<v\left(r_{0}\right)$. Hence there exists a $c>0$ such that $\left(1-w(r)^{2}\right) \geqslant c$ if $r<r_{0}$. Then using Eq. (1.3), we have, for $r<r_{0}$

$$
r A^{\prime}=1-\frac{\left(1-w^{2}\right)^{2}}{r^{2}}-A-\frac{2 v^{2}}{A}<1-\frac{2 v\left(r_{0}\right)^{2}}{A} .
$$

Thus for $r<r_{0}, A^{\prime}(r)<0$ if $A(r) \leqslant 2 v\left(r_{0}\right)^{2}$, so $A(r) \geqslant \min \left(A\left(r_{0}\right), 2 v\left(r_{0}\right)^{2}\right)>0$. Also, Eq. (1.3) implies for $r<r_{0}$

$$
r A^{\prime} \leqslant 1-\frac{\left(1-w^{2}\right)^{2}}{r^{2}} \leqslant 1-\frac{c}{r^{2}}
$$

This implies that $A\left(r_{1}\right)>1$ for some $r_{1}, 0<r_{1}<r_{0}$. This completes the proof of Proposition 2.3.

We see that Theorem 2.1 follows at once from Propositions 2.2 and 2.3. 


\section{REGULAR SOLUTIONS ARE PARTICLELIKE OR BLACK-HOLE SOLUTIONS}

In view of Theorem 2.1 , we may assume that our solution $(A, w)$ satisfies both $w^{2}(r) \leqslant 1$ and $1>A(r) \geqslant 0$, for $r>\rho$, and either $\rho=0$ or $A(\rho)=0$. In this section we will prove that such a solution must be either a particlelike solution, if $\rho=0$, or a black-hole solution, (c.f., Refs. 1-13). We first consider the case where the solution is defined at $r=\rho$, to show the simplification that this assumption makes in the proof. In Theorem 3.4, we achieve the same result but without that assumption.

We shall need the following result, Proposition 3.1, proven in Ref. 12 (in connection with the study of Hawking radiation, cf. Ref. 16, Chap. 14) and Proposition 3.2, proven in Ref. 11.

Proposition 3.1: Let $(A, w)$ be a smooth solution of Eqs. (1.3), (1.4); then $A(r)>0$, if $r>\rho$. [If $A\left(r_{1}\right)=0$ for $r_{1}>\rho$, then $A^{\prime}\left(r_{1}\right)=0$ and so by Ref. $12,(A, w)$ is the extreme Reissner-Nordström solution, $A(r)=((r-1) / r)^{2}, w(r) \equiv 0$.]

Proposition 3.2: If $A(r)=\left(w(r), w^{\prime}(r), A(r), r\right), a<r \leqslant b$ is an orbit segment in

$$
\Gamma=\left\{\left(w, w^{\prime}, A, r\right): w^{2}<1,1>A>0, r>0,\left(w, w^{\prime}\right) \neq(0,0)\right\}
$$

then $\lim _{r \backslash a}(\theta(b)-\theta(r))<\infty$. [Here $\theta(r)=\operatorname{Tan}^{-1}\left(w^{\prime}(r) / w(r)\right)$.]

We shall now consider the case where the solution $(A, w)$ of Eqs. (1.3), (1.4) is defined at $r=\rho$, and is smooth in the region $r \geqslant \rho$.

Theorem 3.3: Let $(A(r), w(r))$ be a regular solution of Eqs. (1.3), (1.4) defined on (its maximum domain of definition) $r \geqslant \rho$ where $(A, w) \in C^{2+\varepsilon} \times C^{2+\varepsilon}(0<\varepsilon<1)$ in the region $r \geqslant \rho$. Then Eqs. (1.9)-(1.11) hold, and $(A, w)$ is either a particlelike solution $\left[\rho=0, w^{2}(0)=1, w^{\prime}(0)=0\right.$, $A(0)=1$; see Ref. 9] or a black-hole solution $\left[\rho>0,\left(w(\rho), w^{\prime}(\rho)\right) \in \mathscr{C}_{\rho}\right.$; cf. Eq. (3.3), $A(\rho)=0$; see Ref. 9].

Proof: We may assume that $A(\rho) \leqslant 1$. Moreover, as follows from Theorem 2.1 , no regular solution can leave the region $w^{2}(r) \leqslant 1$. Finally, Proposition 3.1 implies that $A(r)>0$ if $r>p$. Next, from Ref. 10, Corollary 3.4, the solution $(A, w)$ must have finite rotation and so from Ref. 8 , Propositions 2.10 and 2.11, $\left(A(r), w^{2}(r), w^{\prime}(r)\right) \rightarrow(1,1,0)$ as $r \rightarrow \infty$. Now if $\rho>0$, then Eq. (1.4) gives

$$
\phi w^{\prime}+w\left(1-w^{2}\right)=0, \text { at } \quad r=\rho .
$$

If $\phi(\rho)=0$, then from Eq. (1.3), $A^{\prime}(\rho)=0$, so by Proposition $3.1 \rho=1$ and the solution is an extreme Reissner-Nordström solution, which continues as a regular solution for $r<1$; this violates the definition of $\rho(=1$, in this case). Thus $\phi(\rho) \neq 0$, so $(A, w)$ defines a black-hole solution as discussed in Ref. 9, because $(A, w) \in C^{2+\varepsilon}(r \geqslant p) \times C^{2+\varepsilon}(r \geqslant \rho)$, and such black-hole solutions are unique; cf. Ref. 7, Appendix. Next if $\rho=0$, then as $(A, w)$ is smooth for all $r \geqslant 0$, expanding $A$ and $w$ in Taylor polynomials gives

$$
\begin{aligned}
& A(r)=A_{0}+A_{1} r+O\left(r^{2}\right), \\
& w(r)=w_{0}+w_{1} r+O\left(r^{2}\right)
\end{aligned}
$$

and we easily obtain from Eqs. (1.3) and (1.4), that $w_{0}^{2}=1, w_{1}=0$, and $A_{0}=1$. That is $A(0)=1$, $w^{2}(0)=1, w^{\prime}(0)=0$, so the solution is a regular particlelike solution as discussed in Ref. 8 , by virtue of the uniqueness theorem for differentiable solutions. The proof of Theorem 3.3 is complete.

In the next theorems (Theorems 3.4 and 3.7) we eliminate the assumption that our solution is defined and smooth at $r=\rho$. For $\rho>0$, we show in Theorem 3.4 that $\lim _{r \backslash \rho}\left(w(r), w^{\prime}(r)\right)=\left(\bar{w}, \bar{w}^{\prime}\right)$ exists, and that $\left(\bar{w}, \bar{w}^{\prime}\right)$ lies on $\mathscr{E}_{\rho}$. In fact, more is true, namely, in the Appendix we prove that $(A, w) \in C^{2+\varepsilon}(r \geqslant \rho) \times C^{2+\varepsilon}(r \geqslant \rho)$, so that $(A, w)$ is analytic at $r=\rho$ and thus must be one of the solutions obtained in Ref. 9. 
For $\rho=0$, we prove in Theorem 3.7, that $A, w$, and $w^{\prime}$ are all defined at $r=0$, and that both $A$ and $w$ are in the class $C^{l}(r \geqslant 0)$. But unlike the black-hole casc, we are unable to prove that $A$ and $w$ have higher differentiability.

We now turn to the more difficult case where our regular solution may not be defined at $r=\rho \geqslant 0$.

Assume $\rho>0$; then as $A(r)=1-(\mu(r) / r)$, and $\mu$ is monotone [ $\mu^{\prime}>0$; cf. Ref. 7, Eq. (2.14)], and $\mu(r)>0$, it follows that $\lim _{r \backslash \rho} \mu(r)$ exists so $\lim _{r \backslash \rho} A(r)$ exists. This limit must be zero, or else the solution can be continued for $r<\rho$; (cf. Ref. 7). Thus we may assume

$$
\lim _{r \searrow \rho} A(r)=0, \text { if } \rho>0 \text {. }
$$

We shall show below that $\lim _{r \backslash \rho}\left(w(r), w^{\prime}(r), \Phi(r)\right)=\left(\bar{w}, \bar{w}^{\prime}, \bar{\Phi}\right)$ exists, and that

$$
\bar{\Phi} \bar{w}^{\prime}+\bar{w}\left(1-\bar{w}^{2}\right)=0 .
$$

Then defining $w(\rho)=\bar{w}, w^{\prime}(\rho)=\bar{w}^{\prime}(\rho)=\bar{w}^{\prime}, A(\rho)=0,\left(w(\rho), w^{\prime}(\rho)\right)$ lies on the curve $\mathscr{C}_{\rho}$ defined by (cf. Ref. 9)

$$
\mathscr{C}_{\rho}=\left\{\left(w, w^{\prime}\right): \Phi(\rho, w, 0) w^{\prime}+w\left(1-w^{2}\right)=0\right\},
$$

where we are using the notation $\Phi(r, w, A)=r-r A-\left(1-w^{2}\right) / r$.

Theorem 3.4: Let $(A(r), w(r))$ be a regular solution of Eqs. (1.3), (1.4) with $\rho>0$. Then $w$ and $w^{\prime}$ have limits $w(\rho)$ and $w^{\prime}(\rho)$ as $r \searrow \rho$, and $\left(w(\rho), w^{\prime}(\rho)\right)$ lies on $\mathscr{C}_{\rho}$.

Proof: From Proposition 3.2, $\lim _{r \backslash \rho} \operatorname{Tan}^{-1}\left(w^{\prime}(r) / w(r)\right)$ must be bounded. Thus for $r$ near $\rho$, $r>\rho,\left(w(r), w^{\prime}(r)\right)$ must lie in one of the four quadrants $Q_{i}, i=1, \ldots, 4$ in the $w-w^{\prime}$ plane. Hence by symmetry, we assume that for $r$ near $\rho,\left(w(r), w^{\prime}(r)\right)$ lies either in $\tilde{Q}_{1}=\left\{\left(w, w^{\prime}\right): w \geqslant 0, w^{\prime} \geqslant 0\right\}$, or in $\bar{Q}_{2}=\left\{\left(w, w^{\prime}\right): w \leqslant 0, w^{\prime} \geqslant 0\right\}$. Note that since $w^{\prime}$ is of one sign near $r=\rho, w$ has a limit as $r \searrow \rho$; call this limit $w(\rho)$.

The proof of this theorem will follow from a few lemmas, the first one shows that $w(\rho)$ must satisfy $-1 \leqslant w(\rho)<0$.

Lemma 3.5: $\left(w(\rho), w^{\prime}(\rho)\right)$ cannot lie in $\bar{Q}_{\mathrm{I}}$.

Proof: Suppose that $\left(w(\rho), w^{\prime}(\rho)\right)$ lies in $\bar{Q}_{1}$. If $v=A w^{\prime}$, then $v$ satisfies the equation

$$
v^{\prime}+\frac{2 w^{\prime 2} v}{r}+\frac{w\left(1-w^{2}\right)}{r^{2}}=0
$$

so in $\bar{Q}_{1}, v^{\prime} \leqslant 0$. Thus if $r_{0}>\rho, v(\rho) \geqslant v\left(r_{0}\right)$ so $\left(A w^{\prime}\right)(\rho) \geqslant v\left(r_{0}\right) \geqslant 0$. Since $A(\rho)=0$, we see that $\lim _{r \searrow \rho} w^{\prime}(r)=\infty$. Thus

$$
\lim _{r \searrow \rho}\left(A w^{\prime 2}\right)(r)=\lim _{r \searrow \rho} w^{\prime}(r) v(r) \geqslant\left(\lim _{r \searrow \rho} w^{\prime}(r)\right) v\left(r_{0}\right)
$$

and hence $\left(A w^{\prime 2}\right)(r) \rightarrow \infty$ as $r \searrow \rho$. Then from Eq. (3.2), we have, for $r>\rho, r$ near $\rho$

$$
A^{\prime}(r)=\frac{\phi}{r^{2}}-\frac{2 A w^{\prime 2}}{r}=\frac{r(1-A)}{r^{2}}-\frac{\left(1-w^{2}\right)^{2}}{r^{3}}-\frac{2 A w^{\prime 2}}{r} \leqslant \frac{1}{r}\left(1-2 A w^{\prime 2}\right)<0 .
$$

Thus for $r$ near $\rho, r>\rho$, we have, for some intermediate point $\xi$

$$
A(r)=A(r)-A(\rho)=A^{\prime}(\xi)(r-\rho)<0
$$

and this is impossible. This contradiction establishes the lemma.

Thus, in what follows, we may assume that $-1 \leqslant w(\rho)<0$. We now have the following lemma. 
Lemma 3.6: If $w^{\prime}(r)$ is bounded near $r=\rho$, then $\phi(\rho)>0$, and $\left(w(\rho), w^{\prime}(\rho)\right) \in \mathscr{C}_{\rho}$.

Proof: Since $w$ has a limit as $r \searrow \rho, \phi(r)=r(1-A)-\left(1-w^{2}\right)^{2} / r$ has a limit as $r \searrow \rho$; call this limit $\Phi(\rho)$. As above, let $v=A w^{\prime}$; note that since $w^{\prime}$ is bounded near $r=\rho, \lim _{r \backslash \rho} v(r)=0$. Then using L'hôpital's rule, together with Eqs. (3.2) and (3.4), we have

$$
\begin{aligned}
\lim _{r \searrow \rho} w^{\prime}(r)=\lim _{r \searrow \rho} \frac{v(r)}{A(r)} & =\lim _{r \searrow \rho}\left[\frac{-2 A w^{\prime 2}}{r}-\frac{w\left(1-w^{2}\right)}{r^{2}}\right] /\left[\frac{\phi}{r^{2}}-\frac{2 A w^{\prime 2}}{r}\right] \\
& =\lim _{r \searrow \rho} \frac{-2 A w^{\prime 2} r-w\left(1-w^{2}\right)}{\phi-2 A w^{\prime 2} r}=\frac{-w(\rho)\left(1-w^{2}(\rho)\right)}{\phi(\rho)} .
\end{aligned}
$$

If $\phi(\rho)=0$, then since $\rho>0$, and $w^{2}(\rho) \neq 1$, we obtain the contradiction that $w^{\prime}$ is unbounded near $r=\rho$. Thus $\phi(\rho)>0$, and at $\rho, w^{\prime}(\rho)=\lim _{r \searrow \rho} w^{\prime}(r)$ exists, and $\phi(\rho) w^{\prime}(\rho)-w(\rho)\left(1-w^{2}(\rho)\right)=0$; this proves the lemma.

We now eliminate the remaining case where $\rho>0$ and $w^{\prime}$ is unbounded near $r=\rho$. Before proceeding, we note that

$$
A(\rho)=0 \quad \text { implies } \underset{r \searrow \rho}{\varlimsup_{x \rightarrow}} A^{\prime}(r) \geqslant 0
$$

[Indeed, if this were not so, then for $r>\rho, r$ near $\rho$, we obtain the contradiction $0<A(r)=A(r)-A(\rho)=A^{\prime}(\xi)(r-\rho)<0$.] It then follows from Eq. (1.3) that

$$
0 \leqslant \varlimsup_{r \searrow \rho}\left[\frac{\Phi(r)}{r}-2\left(A w^{\prime 2}\right)(r)\right]=\varlimsup_{r \backslash \rho}\left[\frac{\Phi(\rho)}{\rho}-2\left(A w^{\prime 2}\right)(r)\right]
$$

so that

$$
0 \leqslant \frac{\lim 2}{r \searrow \rho}\left(A w^{\prime 2}\right)(r) \leqslant \frac{\Phi(\rho)}{\rho} .
$$

In view of Lemma 3.5, we may assume that $w(\rho)$ satisfies $-1 \leqslant w(\rho)<0$. We divide the proof into two subcases: $\phi(\rho) \neq 0$, and $\phi(\rho)=0$. [Note that $\phi(\rho)$ is finite.]

Case 1: $\phi(\rho) \neq 0\left[A(\rho)=0, \rho>0, w^{\prime}\right.$ unbounded near $\left.r=\rho\right]$.

If $\phi(\rho)<0$, then for $r$ near $\rho, r>\rho$

$$
A^{\prime}(r)=\frac{\phi}{r^{2}}-\frac{2 A w^{\prime 2}}{r}<0
$$

and this contradicts Eq. (3.5). We may thus assume $\phi(\rho)>0$.

We first claim that $w^{\prime}$ increases monotonically to $+\infty$. Indeed, if this were not true, then $w^{\prime}$ as unbounded near $r=\rho$, we could find a sequence $r_{n} \searrow \rho$, with $w^{\prime}\left(r_{n}\right)>n$ and $w^{\prime \prime}\left(r_{n}\right)=0$. Then from Eq. (2.1), we get

$$
r_{n}^{2} A\left(r_{n}\right) w^{\prime \prime}\left(r_{n}\right)+\phi\left(r_{n}\right) w^{\prime}\left(r_{n}\right)+w\left(r_{n}\right)\left(1-w^{2}\left(r_{n}\right)\right)=0 .
$$

But this cannot hold for large $n$; this establishes our claim. Next, defining $f=A w^{\prime 2}$, then $f$ satisfies the equation

$$
r^{2} f^{\prime}+(2 r f+\Phi) w^{\prime 2}+4 w\left(1-w^{2}\right) w^{\prime}=0
$$


Since $\Phi(\rho)>0$, we see that since $w^{\prime}(r) \rightarrow \infty$ as $r \searrow \rho$, we must have $f^{\prime}(r)<0$ for $r$ near $\rho$, so $\lim _{r \backslash \rho} f(r)=L>0$ exists. Then $\left(w^{\prime 2} v\right)(r)=\left(w^{\prime} f\right)(r) \rightarrow \infty$ as $r \searrow \rho$, so, [cf. Eq. (3.4)], $v^{\prime}(r) \rightarrow-\infty$ as $r \searrow \rho$. Hence $v(\rho)>v\left(r_{1}\right)$ for some $r_{1}$ near $\rho, r_{1}>\rho$. Then for $r$ near $\rho, r>\rho$, $\left(A w^{\prime 2}\right)(r)=v(r) w^{\prime}(r) \geqslant v(\rho) w^{\prime}(r)$, so $\left(A w^{\prime 2}\right)(r) \rightarrow \infty$ as $r \searrow \rho$. This contradicts Eq. (3.6). Thus Case 1 cannot occur.

We now consider

Case 2: $\phi(\rho)=0\left[A(\rho)=0, \rho>0, w^{\prime}\right.$ unbounded near $\left.r=\rho\right]$.

In this case, we claim that

$$
\lim _{r \searrow \rho}\left(A w^{\prime 2}\right)(r)=0
$$

Indeed, if as above, $f=A w^{\prime 2}$, then from Eq. (3.6), $\lim _{r \backslash \rho} f(r)=0$.

Thus to show $A w^{\prime 2} \rightarrow 0$, it suffices to show that $\overline{\lim }_{r \backslash \rho} f(r)=0$. Now, if $\overline{\lim } f(r)$ $=2 \eta>0$, then we can find points $r_{n} \searrow 0$ such that $f\left(r_{n}\right)=\eta$ and $f^{\prime}\left(r_{n}\right)>0$. Since $f\left(r_{n}\right)=\eta$ and $A\left(r_{n}\right) \rightarrow 0$, we see that $w^{\prime}\left(r_{n}\right) \rightarrow \infty$. Using Eq. (3.7) at $r=r_{n}$, we see (as before) that for large $n$, the left-hand side is positive, and this gives the desired contradiction. Thus $A w^{\prime 2} \rightarrow 0$ as $r \searrow \rho$.

We next show that

$$
\lim _{r \backslash \rho} w^{\prime}(r)=\infty
$$

To sec this, note that Eq. (3.8) means: given any $M>0$, there is a $\delta>0$ such that if $\rho<r<r+\delta$, then $w^{\prime}(r)>M$. Thus, if Eq. (3.8) fails, then (since we are in the case where $w^{\prime}$ is unbounded near $r=\rho)$ we can find sequences $s_{n} \searrow \rho, t_{n} \searrow \rho$ such that $w^{\prime}\left(s_{n}\right)<n, w^{\prime}\left(t_{n}\right)>n$, with $s_{n}>t_{n}>s_{n+1}$. Since $\max w^{\prime}(r)>n$ on $s_{n+1} \leqslant r \leqslant s_{n}$, there is a point $r_{n}$ in this interval where $w^{\prime \prime}\left(r_{n}\right)=0$, $w^{\prime \prime \prime}\left(r_{n}\right) \leqslant 0$. But from Eq. $(2.1)$

$$
\begin{aligned}
\left.r^{2} A w^{\prime \prime \prime}\right|_{r_{n}} & =\left.\left[-\phi^{\prime}-\left(1-3 w^{2}\right)\right] w^{\prime}\right|_{r_{n}} \\
& =-w^{\prime}\left(r_{n}\right)\left[\frac{2\left(1-w^{2}\right)}{r_{n}^{2}}+2 A w^{\prime 2}+\frac{4 w\left(1-w^{2}\right) w^{\prime}}{r_{n}}+1-3 w^{2}\right]_{r_{n}} .
\end{aligned}
$$

Since $w\left(1-w^{2}\right) \neq 0$ for $r=\rho$ [because $\phi(\rho)=0$ implies that $w^{2}(\rho) \neq 1$, and Lemma 3.5 implies $w(\rho) \neq 0]$ the term $4 w\left(1-w^{2}\right) w^{\prime} / r_{n}$ tends to $-\infty$ as $n \rightarrow \infty$, so that for large $n, r_{n}^{2}\left(A w^{\prime \prime \prime}\right)\left(r_{n}\right)>0$, and this is a contradiction; thus Eq. (3.8) holds.

Now choose $\delta>0$ such that if $\rho<r<\rho+\delta$

$$
\phi^{\prime}(r)=\frac{2\left(1-w^{2}\right)^{2}}{r^{2}}+2 A w^{\prime 2}+\frac{4 w\left(1-w^{2}\right) w^{\prime}}{r}<0 ;
$$

this can be done since $w(\rho)\left(1-w^{2}(\rho)\right) \neq 0$. Since $\phi(\rho)=0$, Eq. (3.9) shows that $\phi(r)<0$ for $r$ near $\rho, r>\rho$ and we again obtain the contradiction

$$
A^{\prime}(r)=\frac{\phi}{r^{2}}-2 A w^{\prime 2}<0
$$

for $r$ near $\rho$ [cf. Eq. (3.5)]. Thus Case 2 cannot occur, so that if $\rho>0$ and $A(\rho)=0$, then the crash occurs on $\mathscr{E}_{\rho}$. This completes the proof of Theorem 3.4.

We now consider the case $\rho=0$. In this case we only assume that

$$
\varlimsup_{r \searrow 0} A(r) \leqslant 1
$$


Note that in view of Propositions 2.1 and 2.6, we may suppose that $w^{2}(r) \leqslant 1$ for all $r \geqslant 0$. Moreover, exactly as in the case $\rho>0$, it follows from Proposition 3.2, that $\lim _{r \backslash 0} \operatorname{Tan}^{-1}\left(w^{\prime}(r) / w(r)\right)$ is bounded, so for $r$ near $0,\left(w(r), w^{\prime}(r)\right)$ must lie in one of the four quadrants $Q_{i}(i=1-4)$ in the $w-w^{\prime}$ plane; by symmetry we can assume that for $r$ near 0 , $\left(w(r), w^{\prime}(r)\right)$ lies in $\bar{Q}_{1} \cup \bar{Q}_{2}$. Again since $w^{\prime}$ is of one sign near $r=0, \lim _{r \backslash 0} w(r)$ exists; call this limit $\bar{w}$. Finally, as one can easily check, the proof of Lemma 3.5 is valid for $\rho=0$. Thus we may assume that

$$
-1 \leqslant \bar{w}<0
$$

We now have

Theorem 3.7: Let $(A(r), w(r))$ be a regular solution of Eqs. (1.3), (1.4) defined in the region $r>0$. Then $\lim _{r \backslash 0}\left(A(r), w^{2}(r), w^{\prime}(r)\right)$ exists and equals $(1,1,0)$ [as is true of particlelike solutions of Eqs. (1.3), (1.4); cf. Ref. 8].

The proof of this theorem will be divided into a series of lemmas, the first of which is Lemma 3.8: $\lim _{r \backslash 0} w^{2}(r)=1$.

Proof: Assume $\bar{w}^{2} \neq 1$. Choose $\bar{r}>0$ such that $\bar{r}^{2}<\left(1-\bar{w}^{2}\right)^{2}$ and let $0<r<\bar{r}$. Then from Eq. (1.3), for small $r$

$$
r A^{\prime}=1-A-\frac{\left(1-w^{2}\right)}{r^{2}}-2 A w^{\prime 2} \leqslant \frac{-\bar{r}^{2}}{r^{2}}
$$

and this easily implies that $A(r) \rightarrow \infty$ as $r \searrow 0$. This contradiction establishes the result.

We now consider the following two functions which were introduced in Ref. 7, namely

$$
g(r)=2 r^{2}-\left(1-w^{2}(r)\right)
$$

and

$$
k(r)=A(r)-w^{2}(r)
$$

Concerning these functions, we have the following result which follows from (Ref. 7; Lemmas 4.1 and 4.2):

Lemma 3.9: Let $(A(r), w(r))$ be a solution of Eqs. (1.3), (1.4) which is defined for all $r>\alpha$, and satisfies $w^{2}(r) \leqslant 1$. Then we cannot simultaneously have the following three inequalities holding at any $r_{1}>\alpha$ :

$$
g\left(r_{1}\right)<0, \quad g^{\prime}\left(r_{1}\right)<0, \quad k\left(r_{1}\right)<0 .
$$

We can now state

Lemma 3.10: $\varlimsup_{\lim _{r} \backslash 0} A(r)=1$.

Proof: We must show that $\varlimsup_{r \backslash 0} A(r)<1$ is impossible.

Thus, suppose that $\varlimsup_{\lim _{r} \backslash 0} A(r)<1$, and choose $C$ such that

$$
\varlimsup_{\lim _{r \backslash 0}} A(r)<C<1 .
$$

Then for small $r$, say $0<r<\varepsilon, A(r)<C$ so

$$
\phi(r)=r(1-A)-\frac{\left(1-w^{2}\right)^{2}}{r} \geqslant r(1-C)-\frac{\left(1-w^{2}\right)^{2}}{r} .
$$

Now with $g$ and $k$ as defined in Eqs. (3.13) and (3.14), respectively, we have $g(0)=0$ and $k(0)<0$. It thus follows from Lemma 3.8 , that there is an $\varepsilon>0$ such that 


$$
g(r)=2 r^{2}-\left(1-w^{2}(r)\right)>0, \quad \text { if } \quad 0<r<\varepsilon .
$$

[Indeed, if there were a sequence $r_{n} \searrow 0$ for which $g\left(r_{n}\right)<0$, then by the mean-value theorem we could find $s_{n} \searrow 0$ such that both $g\left(s_{n}\right)<0$ and $g^{\prime}\left(s_{n}\right)<0$, and this violates Lemma 3.9 since $k$ is negative near $r=0$.] Thus $\left(1-w^{2}(r)\right) / 2 r^{2}<1$ so $\left(1-w^{2}\right)^{2} / r<2 r\left(1-w^{2}\right)$, if $0<r<\varepsilon$. Using this in Eq. (3.16), we obtain

$$
\phi(r) \geqslant r(1-C)-2 r\left(1-w^{2}\right)=r\left(1-C-2\left(1-w^{2}\right)\right)
$$

so that if $r$ is near zero, then because $w^{2} \rightarrow 1$

$$
\phi(r) \geqslant C_{1} r, \quad C_{1}>0
$$

Now in Eq. (2.1), if $r$ is near 0

$$
r^{2} A w^{\prime \prime}=-\phi w^{\prime}-w\left(1-w^{2}\right) \leqslant-C_{1} r w^{\prime}-w\left(1-w^{2}\right) \leqslant-C_{1} r w^{\prime}+2 r^{2},
$$

where we have used Eq. (3.17) and the fact that $w(r)<0$ for $r$ near 0; cf. Eq. (3.12). Thus for $r$ near 0 , say, $0<r<r_{1}$

$$
r^{2} A w^{\prime \prime} \leqslant r\left(2 r-C_{1} w^{\prime}\right)
$$

We now consider two cases.

Case 1: $0 \leqslant w^{\prime}(r) \leqslant 2 r / C_{1}$, if $0<r<r_{1}$.

In this case $w^{\prime}(r) \rightarrow 0$, so Eq. (1.3) gives, for $0<r<r_{1}$

$$
r A^{\prime}=-\left(1+2 w^{\prime 2}\right) A+1-\frac{\left(1-w^{2}\right)^{2}}{r^{2}} \geqslant \delta
$$

for some constant $\delta>0$ in view of Eqs. (3.15) and (3.17). Integrating the inequality $A^{\prime} \geqslant \delta / r$ from $r<r_{1}$ to $r_{1}$ gives $A\left(r_{1}\right)-A(r) \geqslant \delta \log \left(r_{1} / r\right)$ so $A(r) \rightarrow-\infty$ as $r \searrow 0$. Thus $A(r)=0$ for some $r>0$, and this is a contradiction.

Case 2: $w^{\prime}(\bar{r})>2 \bar{r} / C_{1}$, for some $\bar{r}, 0<\bar{r}<r_{1}$.

In this case, Eq. (3.18) shows that $w^{\prime \prime}(\bar{r})<0$, so if $0<r<\bar{r}$ and $r$ is near $\bar{r}$, $w^{\prime}(r)>w^{\prime}(\bar{r})>2 \bar{r} / C_{1}>2 r / C_{1}$ so Eq. (3.18) implies $w^{\prime \prime}(r)<0$. It follows that for $0<r<\bar{r}$, $w^{\prime}(r)>w^{\prime}(\bar{r})>2 \bar{r} / C_{1}$; thus

$$
\underline{\lim } w^{\prime}(r) \geqslant 2 \bar{r} / C_{1}>0
$$

Now [cf. Eqs. (3.14) and (3.15)] $k(r)=A(r)-w^{2}(r)<0$ for $r$ near 0 , and since $w(r) \rightarrow-1$ as $r \searrow 0$ (by Lemma 3.8), we can take $r$ so close to zero that $-1 \leqslant w(r)<-\frac{1}{2}$. Then for these $r$, Eq. (3.20) gives

$$
g^{\prime}(r)=2\left(2 r+w w^{\prime}\right) \leqslant 2\left(2 r+w(r) \frac{\lim }{r \searrow 0} w^{\prime}(r)\right) \leqslant 2\left(2 r-\frac{1}{2} \underset{r \backslash 0}{\lim } w^{\prime}(r)\right)<0
$$

for small $r$. Since $g(0)=0$, we see that this contradicts Lemma 3.9. This completes this proof of Lemma 3.10.

We next prove

Lemma 3.11: $\lim _{r \backslash 0} w^{\prime}(r)=0$.

Proof: Suppose that $\lim _{r \backslash 0} w^{\prime}(r)=\eta>0$. Choose $C>0$ such that $\left(1+2 \eta^{2}\right)^{-1}<C<1$. From Lemma 3.10, we can find an $r_{1}>0$ such that $A\left(r_{1}\right)>C$. Using Eq. (1.3), we have, at $r=r_{1}$ 
$r_{1} A^{\prime}\left(r_{1}\right)=-\left(1+2 w^{\prime 2}\right) A\left(r_{1}\right)+1-\frac{\left(1-w^{2}\right)^{2}}{r_{1}^{2}} \leqslant 1-\left(1+2 \eta^{2}\right) A\left(r_{1}\right)<1-\left(1+2 \eta^{2}\right) C \equiv-\delta^{2}<0$

Thus if $0<r<r_{1}, A(r)>A\left(r_{1}\right)>C$, so $r A^{\prime}(r) \leqslant-\delta^{2}$. As we have seen earlier, this implies the contradiction $A(r) \rightarrow \infty$ as $r \searrow 0$.

Now in view of the last two results, the proof of Theorem 3.7 will be completed if we can eliminate the following three cases.

Case I:

Case II:

Case III:

$$
\lim _{r \searrow 0} A(r)=1, \text { and } \underset{\lim _{r \searrow 0}}{ } w^{\prime}(r)>0
$$

$$
\lim _{r \backslash 0} A(r)<1, \quad \text { and } \varlimsup_{r \searrow 0} w^{\prime}(r)=0
$$

$$
\lim _{r \backslash 0} A(r)<1, \text { and } \overline{\lim }_{r \backslash 0} w^{\prime}(r)>0
$$

[Thus if Cases I-III are eliminated, then we must have both $\lim A(r)=1$ and $\overline{\lim } w^{\prime}(r)$ $=0$ so Lemmas 3.10 and 3.11 imply $A(r) \rightarrow 1$ and $w^{\prime}(r) \rightarrow 0$ as $\backslash 0$.]

We begin with the following lemma.

Lemma 3.12: If $\overline{\lim }_{r \backslash 0} w^{\prime}(r)>0$, then both of the following hold:

$$
\frac{d}{d r}\left(\frac{1-w^{2}(r)}{r}\right)>0
$$

and

$$
\lim _{r \searrow 0} \frac{1-w^{2}(r)}{r}=0
$$

Proof: Using Lemma 3.11, we can find points $r_{n} \searrow 0$ such that

$$
w^{\prime \prime}\left(r_{n}\right)=0, \text { and } w^{\prime}\left(r_{n}\right)<1 \text {. }
$$

Using Eq. (2.1)

$$
0=-r_{n}^{2} A\left(r_{n}\right) w^{\prime \prime}\left(r_{n}\right)=\phi w^{\prime}+\left.w\left(1-w^{2}\right)\right|_{r=r_{n}}
$$

so that at $r_{n}$

$$
\frac{\phi w^{\prime}}{r_{n}}+\frac{w\left(1-w^{2}\right)}{r_{n}}=0
$$

or

$$
(1-A) w^{\prime}-\frac{\left(1-w^{2}\right)^{2}}{r_{n}^{2}} w^{\prime}+\frac{w\left(1-w^{2}\right)}{r_{n}}=0 .
$$

We consider the left side of Eq. (3.23) as a quadratic function in $\left(1-w^{2}\right) / r_{n}$. Solving for the roots, we find 


$$
\frac{1-w^{2}\left(r_{n}\right)}{r_{n}} \leqslant\left.\frac{w+\sqrt{w^{2}+r_{n}(1-A) w^{\prime 2}}}{2 w^{\prime}}\right|_{r=r_{n}} .
$$

Now consider the right-hand side of Eq. (3.24) as an abstract function of a variable $w^{\prime}$ ( $w$, $r$, and $A$ are regarded as fixed)

$$
\psi\left(w^{\prime}\right)=\frac{w+\sqrt{w^{2}+r(1-A) w^{\prime 2}}}{2 w^{\prime}} .
$$

Concerning this function, easy calculations show that $\psi^{\prime}>0$. This implies that $\psi\left(w^{\prime}\right)$ $<\lim _{w^{\prime} \rightarrow \infty} \psi\left(w^{\prime}\right)=\frac{1}{2} \sqrt{r(1-A)}$. Furthermore, we claim that for $r$ near 0 [and hence $w(r)$ near $-1]$

$$
\psi\left(w^{\prime}\right)<w^{\prime}
$$

[Indeed, if $\psi\left(w^{\prime}\right) \geqslant w^{\prime}$, then $\sqrt{w^{2}+r(1-A) w^{\prime 2}} \geqslant 2 w^{\prime 2}-w$, so squaring and simplifying gives

$$
0 \geqslant 4 w^{\prime 2}(r)+[-4 w(r)-r(1-A(r))] .
$$

But since $w(r) \rightarrow-1$ and $r(1-A(r)) \rightarrow 0$ as $r \searrow 0$, we obtain a contradiction.] Then using Eq. (3.25) in Eq. (3.24), we get, for large $n$

$$
\left(\frac{1-w^{2}\left(r_{n}\right)}{r_{n}}\right) \leqslant w^{\prime}\left(r_{n}\right)
$$

Now let

$$
\theta(r)=1-w^{2}(r)+2 r\left(w w^{\prime}\right)(r)
$$

then

$$
\frac{d}{d r}\left(\frac{1-w^{2}(r)}{r}\right)=\frac{-\theta(r)}{r^{2}}
$$

Thus to prove Eq. (3.20), we must show

$$
\theta(r)<0
$$

if $r$ is close to zero. Now taking $n$ so large that $-1<w\left(r_{n}\right)<-\frac{1}{2}$ (cf. Lemma 3.8), we have, from Eq. (3.26),

$$
\theta\left(r_{n}\right)=r_{n}\left[\frac{1-w^{2}\left(r_{n}\right)}{r_{n}}+2\left(w w^{\prime}\right)\left(r_{n}\right)\right] \leqslant r_{n}\left[w^{\prime}\left(r_{n}\right)+2\left(w w^{\prime}\right)\left(r_{n}\right)\right]=r_{n} w_{n}^{\prime}\left(r_{n}\right)\left[1+2 w\left(r_{n}\right)\right]
$$

thus

$$
\theta\left(r_{n}\right)<0 \text {, if } n \text { is large. }
$$

Next, we claim that if $r$ is so close to zero that $0<r<\frac{1}{2},-1<w(r)<-\frac{1}{2}$, and $r+w(r)<0$, we have

$$
\theta^{\prime}(r)<0, \quad \text { if } \quad \theta(r)=0
$$

To see this, we compute 


$$
\begin{aligned}
\left.\theta^{\prime}(r)\right|_{\theta=0} & =2 r\left[w^{\prime 2}-\frac{w^{2}\left(1-w^{2}\right)}{r^{2} A}-\frac{\phi w w^{\prime}}{r^{2} A}\right] \\
& =\frac{2}{r A}\left[r^{2} A w^{\prime 2}-w^{2}\left(1-w^{2}\right)-r w w^{\prime}+r A w w^{\prime}+\frac{\left(1-w^{2}\right)}{r^{2}} w w^{\prime}\right] \\
& =\frac{2}{r A}\left[r^{2} A w^{\prime 2}+2 r w^{3} w^{\prime}-r w w^{\prime}+r A w w^{\prime}+4 r w^{3} w^{\prime 3}\right] \\
& =\frac{2 w^{\prime}}{A}\left[r A w^{\prime}+2 w^{3}-w+A w+4 w^{3} w^{\prime 2}\right] .
\end{aligned}
$$

Now if $w^{\prime} \leqslant 1$, then

$$
r A w^{\prime}+2 w^{3}-w+A w+4 w^{3} w^{\prime 2} \leqslant\left(r A w^{\prime}+A w\right)<A(r+w)<0
$$

thus Eq. (3.30) holds. On the other hand, if $w^{\prime} \geqslant 1$

$$
\begin{aligned}
r A w^{\prime}+2 w^{3}-w+A w+4 w^{3} w^{\prime 2} & =\left(r A w^{\prime}+4 w^{3} w^{\prime 2}\right)+w\left(2 w^{2}-1\right)+A w \\
& <r w^{\prime}+4 w^{3} w^{\prime} \\
& =w^{\prime}\left(r+4 w^{3}\right) \\
& <w^{\prime}\left(r-\frac{1}{2}\right) \\
& <0
\end{aligned}
$$

thus Eq. (3.30) holds. Then Eq. (3.28) follows from Eq. (3.30), and this proves Eq. (3.20).

Finally, Eq. (3.20) implies that for $r$ near 0

$$
\frac{1-w^{2}(r)}{r}<-2 w(r) w^{\prime}(r)<2 w^{\prime}(r) .
$$

Using Lemma 3.11, we have $\lim _{r \backslash 0}\left(1-w^{2}(r)\right) / r=0$, and in view of Eq. (3.20), we see that Eq. (3.21) holds. This completes the proof of Lemma 3.12 .

Lemma 3.13: Let $v=A w^{\prime}$, then if $v$ has a local maximum at $\bar{r}$

$$
v^{3}(\bar{r}) \leqslant \frac{1-w^{2}(\bar{r})}{2 \bar{r}}
$$

Proof: Since $v^{\prime}(\bar{r})=0$, Eq. (3.4) gives

$$
\frac{-2 w^{\prime 2}(\bar{r}) v(\bar{r})}{\bar{r}}=w(\bar{r})\left(1-w^{2}(\bar{r})\right)
$$

so at $\bar{r}$

$$
v^{3}(\bar{r})=\frac{-w\left(1-w^{2}\right) A^{2}}{2 \bar{r}} \leqslant \frac{1-w^{2}(\bar{r})}{2 \bar{r}}
$$

Lemma 3.14: If $\lim _{r \backslash \infty} A(r)=1$, then $\overline{\lim }_{r \backslash 0} w^{\prime}=0$.

Proof: Assume that 


$$
\overline{\lim }_{r \backslash 0} w^{\prime}(r)>0 \text {. }
$$

Since $\lim _{r \backslash 0} A(r)=1$, Lemma 3.10 implies that $\lim _{r \backslash 0} A(r)=1$.

Now let $v$ have a local maximum at $\bar{r}$; infinitely many such points exist arbitrarily near $r=0$, in view of Eq. (3.32) together with Lemma 3.11. At $\bar{r}$, Eq. (3.31) holds, and in view of Eq. (3.21), this implies $\widetilde{\lim }_{r \backslash 0} v(r)=0$. Since $\lim _{r \backslash 0} A(r)=1$, this contradicts Eq. (3.32).

In view of Lemma 3.14, we see that Case I cannot occur.

We next consider Case II

$$
\lim _{r \searrow 0} A(r)<1 \text { and } \varlimsup_{r \searrow 0} w^{\prime}(r)=0
$$

Using Lemma 3.11, we see that $\lim _{r \backslash 0} w^{\prime}(r)=0$. In view of Lemma 3.10, we can find points $r_{n} \searrow 0$ such that $A^{\prime}\left(r_{n}\right)=0$, and

$$
A\left(r_{n}\right) \rightarrow \frac{\lim }{r \searrow 0} A(r)<1
$$

At these points, Eq. (1.3) implies that

$$
A\left(r_{n}\right)=\left[1-\left(\frac{1-w^{2}\left(r_{n}\right)}{r_{n}}\right)\right] /\left[1+2 w^{\prime 2}\left(r_{n}\right)\right] .
$$

But using L'Hôpital's rule

$$
\lim _{r \searrow 0} \frac{1-w^{2}(r)}{r}=\lim _{r \searrow 0}-2 w(r) w^{\prime}(r)=0 .
$$

Thus Eq. (3.35) implies that $A\left(r_{n}\right) \rightarrow 1$ and this contradicts Eq. (3.34). Thus Case II cannot occur.

We can now eliminate the final case, Case III

$$
\frac{\lim }{r \searrow 0} A(r)<1, \quad \text { and } \quad \frac{\lim }{r \backslash 0} w^{\prime}(r)>0
$$

Using Lemma 3.11, we can find points $\rho_{n} \searrow 0$ such that $w^{\prime \prime}\left(\rho_{n}\right)=0$, and

$$
w^{\prime}\left(\rho_{n}\right) \geqslant \eta>0 \text {. }
$$

From Eq. (2.1), $\phi\left(\rho_{n}\right) w^{\prime}\left(\rho_{n}\right)+w\left(\rho_{n}\right)\left(1-w^{2}\left(\rho_{n}\right)\right)=0$, and as $-1<w\left(\rho_{n}\right)<0$, we see that $\phi\left(\rho_{n}\right) \neq 0$, so at $\rho_{n}$

$$
w^{\prime}\left(\rho_{n}\right)=\frac{-w\left(1-w^{2}\right)}{\phi}=\left[-\frac{\left(1-w^{2}\right)}{r} w\right] /\left[(1-A)-\frac{\left(1-w^{2}\right)^{2}}{r^{2}}\right] .
$$

From Eqs. (3.21) and (3.37), we see that

$$
1-A\left(\rho_{n}\right) \rightarrow 0
$$

Thus for large $n, A\left(\rho_{n}\right)>\frac{1}{2}$. If $v=A w^{\prime}$, then Eq. (3.37) gives

$$
v\left(\rho_{n}\right) \geqslant \eta / 2 \text {. }
$$

Now Lemma 3.11 implies that we can find a sequence $\sigma_{n} \searrow 0$ such that $w^{\prime}\left(\sigma_{n}\right) \rightarrow 0$. (We may assume, by reindexing, if necessary, that $\sigma_{n}>\rho_{n}, n=1,2, \ldots$.) Now Lemma 3.10 gives $v\left(\sigma_{n}\right) \rightarrow 0$, 
and this together with Eq. (3.38) implies that we can find a sequence $r_{n} \rightarrow 0, r_{n} \geqslant \rho_{n+1}$, such that $v$ has a local maximum at $r_{n}$, and $v\left(r_{n}\right) \geqslant v\left(\rho_{n}\right)$. Using Lemma 3.13 together with Eq. (3.21) and Lemma 3.8, we conclude that $v\left(r_{n}\right) \rightarrow 0$, and hence $v\left(\rho_{n}\right) \rightarrow 0$. This contradicts Eq. (3.38) and thus Case III cannot occur.

This completes the proof of Theorem 3.7.

We have shown that any solution with event horizon $\rho>0$ has initial values $\left(w(\rho), w^{\prime}(\rho)\right)$ lying on the curve $\mathscr{C}_{\rho}$. It still remains to be shown that any such solution is one of the black-hole solutions whose existence was first established in Ref. 9; this will be done in the Appendix.

\section{KRUSKAL COORDINATES}

In this section we shall show that for the EYM black holes the singularity at $r=\rho$ can be transformed away by choosing "Kruskal" coordinates (see, e.g., Ref. 15 or 16).

Fix $\rho>0$ and let $\left(A(r), w(r), w^{\prime}(r), r\right)$ be a black-hole solution satisfying Eqs. (1.3), (1.4). That is, $A(\rho)=0$, and $\left(w(\rho), w^{\prime}(\rho)\right)$ lie on the curve

$$
\mathscr{C}_{p}=\left\{\left(w, w^{\prime}\right): \Phi(\rho, w, 0) w^{\prime}+w\left(1-w^{2}\right)=0\right\},
$$

where $\Phi(\rho, w, 0)=\rho-\left(1-w^{2}\right)^{2} / \rho$. As in Ref. 15, we seek a nonsingular transformation $(r, t) \rightarrow(u, v)$ near $r=\rho$ such that in $u-v$ coordinates, the metric (1.1) is of the form

$$
d s^{2}=f(u, v)^{2}\left(d v^{2}-d u^{2}\right)+r^{2} d \Omega^{2}
$$

where $f(u, v) \neq 0$ near $r=\rho$; cf. Ref. 15. Using the (tensorial) transformation rules for the metrics, ${ }^{15}$ we find, as in Ref. 15 , that the following equations must hold:

$$
\begin{gathered}
-T^{2}=f^{2}\left[\left(\frac{\partial v}{\partial t}\right)^{2}-\left(\frac{\partial u}{\partial t}\right)^{2}\right], \\
0=\frac{\partial u}{\partial t} \frac{\partial u}{\partial r}-\frac{\partial v}{\partial t} \frac{\partial v}{\partial r}, \\
A^{-1}=f^{2}\left[\left(\frac{\partial v}{\partial r}\right)^{2}-\left(\frac{\partial u}{\partial r}\right)^{2}\right] .
\end{gathered}
$$

In order to analyze these equations, we define (as in Refs. 7-10), the functions $Q$ and $P$ by

$$
\begin{gathered}
Q^{\prime}=\frac{2 w^{\prime 2}}{r}, \quad Q(\infty)=0, \\
P^{\prime}=\frac{\Phi}{r^{2} A}, \quad P(\infty)=0 .
\end{gathered}
$$

Note that $Q$ and $P$ have convergent integrals since $\lim _{r \rightarrow \infty} r w^{\prime}(r)=0, A(r)>0$ for $r>\rho$, and $\lim _{r \backslash \rho} \Phi(r) / A(r)$ exists and is finite (see Ref. 10, Lemma 4.1). Next we have the formulas

$$
(\ln A)^{\prime}=P^{\prime}-Q^{\prime}, \quad \text { and } \quad\left(\ln T^{-2}\right)=P^{\prime}+Q^{\prime}
$$

so that

$$
A T^{-2}=e^{2 P}, \text { and } A T^{2}=e^{-2 Q}
$$


We define a new variable $\xi$, by [For the Schwarzschild solution, $w(r) \equiv 1, \Phi=2 m$, $P^{\prime}=(2 m / r)(1 /(r-2 m))$, so

$$
P(r)=\int_{r}^{\infty} \frac{2 m}{s}(2 m-s)^{-1} d s=\ln \left(\frac{r-2 m}{r}\right), \quad \xi_{r}=e^{-P}=1+\left(\frac{r}{2 m}-1\right)^{-1},
$$

and

$$
\xi=r+2 m \ln \left(\frac{r}{2 m}-1\right)
$$

cf. Ref. 1.]

$$
\xi_{r}=e^{-P(r)}, \quad \xi(\rho+1)=0
$$

(so $\xi_{r}>0$ and thus the map $r \rightarrow \xi$ is one-one), and set

$$
F(\xi)=(T f)^{-2}
$$

Then Eqs. (4.3)-(4.5) become

$$
\begin{gathered}
\left(\frac{\partial v}{\partial t}\right)^{2}-\left(\frac{\partial u}{\partial t}\right)^{2}=-F(\xi) \\
\left(\frac{\partial u}{\partial t}\right)\left(\frac{\partial u}{\partial \xi}\right)-\left(\frac{\partial v}{\partial t}\right)\left(\frac{\partial v}{\partial \xi}\right)=0 \\
\left(\frac{\partial v}{\partial \xi}\right)^{2}-\left(\frac{\partial u}{\partial \xi}\right)^{2}=F(\xi) .
\end{gathered}
$$

Now from these equations, proceeding in a standard way, as in the Schwarzschild case (see Ref. 15) we obtain

$$
v=e^{\eta \xi} \sinh (\eta t), \quad u=e^{\eta \xi} \cosh (\eta t), \quad F(\xi)=\eta^{2} e^{2 \eta \xi},
$$

where $\eta$ is a constant, still to be chosen. From Eq. (4.11) it follows that

$$
f^{2}(r)=\frac{T^{-2}}{\eta^{2} e^{2 \eta \xi}}
$$

To complete the transition to Kruskal coordinates, we must show two things, namely, for an appropriate choice of $\eta$

$$
f^{2}(r) \neq 0 \quad \text { near } \quad r=\rho
$$

and that the Yang-Mills curvature, $F$, defined by Eq. (1.2) is nonsingular near $r=\rho$. We begin with Eq. (4.13).

Proposition 4.1: The constant $\eta$ can be chosen so that $f(\rho) \neq 0$.

Proof: Using Eq. (4.6), we have from Eq. (4.12)

$$
f^{2}=\frac{A e^{2 Q}}{\eta^{2} e^{2 \eta \xi}}
$$


Then since $w^{\prime}$ is bounded at $r=\rho$, we see that $Q(\rho)>-\infty$; thus from Eq. (4.14) we see that $f^{2}(\rho) \neq 0$ if and only if

$$
\lim _{r \rightarrow \rho} \frac{A(r)}{e^{2 \eta \xi}} \neq 0
$$

Since

$$
A=e^{P-Q}
$$

we have $f(p) \neq 0$ if and only if

$$
P-Q-2 \eta \xi>-\infty \text { at } r=\rho .
$$

Now as $A(\rho)=0$, and $Q(\rho)$ is finite, Eq. (4.16) shows that $P(r) \rightarrow-\infty$ as $r \rightarrow \rho$. It follows that we must show that we can choose $\eta \neq 0$ such that

$$
P-2 \eta \xi \text { is finite at } r=\rho .
$$

Now Eq. (4.18) holds if

$$
P^{\prime}-2 \eta e^{-P} \text { is bounded near } r=\rho .
$$

Since $A^{\prime}(\rho) \neq 0, A(r)=(r-\rho) B(r)$, where $B(\rho) \neq 0$. From Eq. (4.16) $e^{-Q} e^{P}=(r-\rho) B(r) e^{Q(r)}$, so $e^{P}=(r-\rho) B(r) e^{Q(r)} \equiv(r-\rho) C(r), C(\rho) \neq 0$. Thus

$$
\begin{gathered}
P(r)=C(r)+\ln (r-\rho), \quad C(\rho) \neq 0, \\
e^{-P}=e^{-C(r)} /(r-\rho)
\end{gathered}
$$

so

$$
P^{\prime}-2 \eta e^{-P}=\frac{1}{(r-\rho)}\left[1-\frac{2 \eta e^{-Q(r)}}{B(r)}\right]+\frac{C^{\prime}}{C} .
$$

If we choose $\eta$ such that $1=2 \eta e^{-\ell(\rho)} / B(\rho)$, then we see that Eq. (4.19) holds, and thus we have shown that $A^{\prime}(\rho) \neq 0$ implies $f(\rho) \neq 0$.

We now investigate the Yang-Mills field near $r=\rho$, under the above change of coordinates. From Eq. (4.11), we have

$$
\begin{gathered}
v_{\xi}=\eta v, \quad v_{t}=\eta u, \\
u_{\xi}=\eta u, \quad u_{t}=\eta v, \quad \text { and } u^{2}-v^{2}=e^{2 \eta \xi}
\end{gathered}
$$

Now let $T$ and $S$ be the transformations

$$
(r, t) \stackrel{T}{\rightarrow}(\xi, t) \stackrel{s}{\rightarrow}(u, v) .
$$

Then from Eq. (4.22)

$$
d T=\left(\begin{array}{cc}
\xi_{r} & 0 \\
0 & 1
\end{array}\right)=\left(\begin{array}{cc}
e^{-P} & 0 \\
0 & 1
\end{array}\right)=\left(\begin{array}{cc}
\frac{e^{-c(r)}}{r-\rho} & 0 \\
0 & 1
\end{array}\right)
$$




$$
d S=\left(\begin{array}{cc}
\eta u & \eta v \\
\eta v & \eta u
\end{array}\right), \quad(d S)^{-1}=\frac{1}{\eta^{2}\left(u^{2}-v^{2}\right)}\left(\begin{array}{cc}
\eta u & -\eta v \\
-\eta v & \eta u
\end{array}\right)
$$

Then

$$
\left(\begin{array}{cc}
r_{u} & r_{v} \\
t_{u} & t_{v}
\end{array}\right)=(d S d T)^{-1}=(d T)^{-1}(d S)^{-1}=\frac{1}{\eta\left(u^{2}-v^{2}\right)}\left(\begin{array}{cc}
u e^{c}(r-\rho) & -v e^{c}(r-\rho) \\
-v & u
\end{array}\right)
$$

so from Eq. (4.11)

$$
r_{u}=\frac{u e^{c(r)}(r-\rho)}{\eta e^{2 \eta \xi}}=(r-\rho) e^{-\eta \xi} b
$$

where $b=\eta^{-1} e^{c(r)} \cosh (\eta t)$ is bounded near $r=\rho$. Now as $P-2 \eta \xi \equiv B(r)$ is bounded near $r=\rho$, and from Eq. (4.20)

$$
C(r)=\ln (r-\rho)=P=2 \eta \xi+B(r)
$$

we see that

$$
e^{-\eta \varepsilon}=O(r-\rho)^{-1 / 2}
$$

Thus

$$
r_{u}=O(r-\rho)^{1 / 2}, \quad \text { near } \quad r=\rho
$$

and similarly

$$
r_{v}=O(r-\rho)^{1 / 2}, \text { near } \quad r=\rho .
$$

Next, Eqs. (4.23) and (4.11) give

$$
t_{u}=\frac{-v}{\eta\left(u^{2}-v^{2}\right)}=\frac{e^{\eta \xi} \sinh (\eta t)}{e^{2 \eta \xi}}=O\left(e^{-\eta \xi}\right) .
$$

So from Eq. (4.24)

$$
t_{u}=O(r-\rho)^{-1 / 2}
$$

and similarly

$$
t_{v}=O(r-\rho)^{-1 / 2}
$$

We can now see how the Yang-Mills curvature $F$, defined by Eq. (1.2) transforms. First, noting

$$
S \circ T:(r, t, \theta, \phi) \rightarrow(u, v, \theta, \phi)
$$

we may display the curvature two-form as a matrix 


$$
F=\left[\begin{array}{cccc}
0 & 0 & w^{\prime} \tau_{1} & w^{\prime} \sin \theta \tau_{2} \\
0 & 0 & 0 & 0 \\
-w^{\prime} \tau_{1} & 0 & 0 & -\left(1-w^{2}\right) \sin \theta \tau_{3} \\
-w^{\prime} \sin \theta \tau_{2} & 0 & \left(1-w^{2}\right) \sin \theta \tau_{3} & 0
\end{array}\right]
$$

and using the transformation rule for $(0,2)$-tensors, $\tilde{F}_{i j}=F_{k l}\left(\partial x^{k} / \partial z^{i}\right)\left(\partial x^{l} / \partial z^{j}\right),(r, t, \theta, \phi)=x$ $=x(z), \quad z=(u, v, \theta, \phi)$, we have, near $r=\rho, \quad \tilde{F}_{u v}=F_{r t} r_{u} t_{v}+F_{t r} t_{u} r_{v}=O(1)=\tilde{F}_{\theta \psi}$, $\tilde{F}_{u \theta}=F_{r \theta} r_{u}=O(r-\rho)^{1 / 2}=\tilde{F}_{u \phi}=\tilde{F}_{v \theta}=\tilde{F}_{v \phi}$. Thus the transformed curvature two-form is also well-behaved near $r=\rho$. This completes the proof that for black holes, the singularity in the metric at $r=\rho$ can be transformed away by a Kruskal-type coordinate transformation.

\section{ACKNOWLEDGMENTS}

Both authors were supported in part by the NSF, Contract No. DMS-89-05205. J.A.S. was also supported in part by the ONR, Contract No. N0014-94-1-0691.

\section{APPENDIX: UNIQUENESS OF BLACK-HOLE SOLUTIONS}

We shall prove that if $\rho>0$, the regular solutions which we obtained in Sec. III, by "coming back from infinity," are the solutions which were obtained in Ref. 9. For this, it suffices to show that the solutions (with $\rho>0)$ satisfy $(A, w) \in C^{3}(r \geqslant \rho) \times C^{3}(r \geqslant \rho)$. This will imply that these solutions are analytic at $\rho$ (cf. Ref. 9 , Appendix), and hence must agree with the analytic solutions obtained in Ref. 9.

Before giving the proof, we need a lemma. First some notation; we say $f \in C_{0}^{k}$ if $f \in C^{k}$ and $f(0)=0$.

Lemma $A_{l}$ : Let $k \in \mathbf{Z}, k \geqslant 0$, and assume that $\psi \in C^{k}[0, R]$. Then if $n \in \mathbb{Z}, n \geqslant 0$, $\int_{0}^{r} s^{n} \psi(s) d s=r^{n} \xi(r)$, where $\xi \in C_{0}^{k+1}[0, R]$.

Proof: Proof is by induction on $n$. If $n=0, \int_{0}^{r} \psi(s) d s=\xi(r)$ and clearly $\xi \in C_{0}^{k+1}[0, R]$; thus the result holds for $n=0$ and all $k$. Assume now that the result holds for $n-1$ and all $k$; we show it holds for $n$ (and all $k$ ).

Let

$$
I=\int_{0}^{r} s^{n} \psi(s) d s, \quad \text { and } \quad v(r)=\int_{0}^{r} \psi(s) d s
$$

then integrating by parts gives

$$
I=r^{n} v(r)=n \int_{0}^{r} s^{n-1} v(s) d s
$$

Now $v(r) \in C_{0}^{k+1}(0, R)$, and by our induction hypothesis

$$
\int_{0}^{r} s^{n-1} v(s) d s=r^{n-1} \xi(r)=r^{n} \frac{\xi(r)}{r}
$$

where $\xi \in C_{0}^{k+2}(0, R)$. To complete the proof, we show $\xi(r) / r \in C_{0}^{k+1}[0, R]$. For this, it suffices to show $\xi(0)=0=\xi^{\prime}(0)$. Since $\xi \in C_{0}^{k+2}$, we need only show $\xi^{\prime}(0)=0$. We have

$$
\xi^{\prime}(0)=\lim _{r \rightarrow 0} \frac{\xi(r)-\xi(0)}{r-0}=\lim _{r \rightarrow 0} \frac{\xi(r)}{r}=\lim _{r \rightarrow 0} \frac{\int_{0}^{r} s^{n-1} v(s) d s}{r^{n}}=\lim _{r \rightarrow 0} \frac{r^{n-1} v(r)}{n r^{n-1}}=\frac{v(0)}{n}=0 .
$$


We now show that the solutions $(A, w)$ obtained in Sec. III are analytic at $r=0$. In Sec. III we proved that $\lim _{r \backslash \rho}\left(w(r), w^{\prime}(r)\right)=\left(\bar{w}, \bar{w}^{\prime}\right)$, exists, and $\left(\bar{w}, \bar{w}^{\prime}\right)$ lies on $\mathscr{C}_{\rho}$ [cf. Eq. (3.3)]. We extend the definition of $w$ to make $w$ continuous at $r=\rho$. Next

$$
w^{\prime}(\rho)=\lim _{r \searrow \rho} \frac{w(r)-\bar{w}}{r-\rho}=\lim _{r \searrow \rho} w^{\prime}(r)=\bar{w}^{\prime} .
$$

This shows that $w \in C^{1}(r \geqslant \rho)$. Next, define $A(\rho)=0$; this makes $A$ continuous at $\rho$. Also, from Eq. (1.3)

$$
\lim _{r \searrow \rho} \frac{A(r)-A(\rho)}{r-\rho}=\lim _{r \searrow \rho} A^{\prime}(r)=\frac{\Phi(\rho)}{\rho^{2}}
$$

thus defining $A^{\prime}(\rho)=\Phi(\rho) / \rho^{2}$, we see that $A \in C_{0}^{1}(r \geqslant \rho)$. Next we show that $A \in C_{0}^{2}(r \geqslant \rho)$. Since $\Phi=r(1-A)-\left[\left(1-w^{2}\right)^{2} / r\right] \in C^{1}(r \geqslant \rho)$, we see from Eq. (1.3), that $A \in C_{0}^{2}(r \geqslant \rho)$ if $A w^{\prime 2} \in C^{1}(r \geqslant \rho)$. But from Eq. (3.7), we see that $A w^{\prime 2} \in C^{1}(r \geqslant \rho)$. Thus we have proven that

$$
(A, w) \in C_{0}^{2}(r \geqslant \rho) \times C^{1}(r \geqslant \rho) .
$$

Now write

$$
x=r-\rho
$$

then as $A \in C_{0}^{2}(r \geqslant \rho)$, we claim that

$$
A=x B
$$

where

$$
B \in C^{1}(x \geqslant 0), \quad \text { and } \quad B(0)=\frac{\Phi(\rho)}{r^{2}} \neq 0
$$

To see this, note that $\lim _{x \backslash 0}[A(x) / x]=A^{\prime}(0)$, so if $B(x)=A(x) / x$, then defining $B(0)=A^{\prime}(0)$, we see that $B \in C^{0}(x \geqslant 0)$, and from Eq. (1.3), $B(0)=\Phi(\rho) / \rho^{2}$. Now

$$
\begin{aligned}
\lim _{x \backslash 0} \frac{B(x)-A^{\prime}(0)}{x}=\lim _{x \backslash 0} \frac{[A(x) / x]-A^{\prime}(0)}{x}=\lim _{x \backslash 0} \frac{A(x)-x A^{\prime}(0)}{x^{2}} & =\lim _{x \backslash 0} \frac{A^{\prime}(x)-A^{\prime}(0)}{2 x} \\
& =\frac{1}{2} A^{\prime \prime}(0)
\end{aligned}
$$

so $B$ is differentiable at $x=0$, and $B^{\prime}(0)=\frac{1}{2} A^{\prime \prime}(0)$. Also if $x>0$, $B^{\prime}(x)=\left(x A^{\prime}(x)-A(x)\right) / x^{2}=\left(A^{\prime}(x)-[A(x) / x]\right) / x$, so

$$
\lim _{x \searrow 0} B^{\prime}(x)=\lim _{x \searrow 0}\left[\frac{A^{\prime}(x)-A^{\prime}(0)}{x}+\frac{A^{\prime}(0)-[A(x) / x]}{x}\right]=A^{\prime \prime}(0)-\frac{1}{2} A^{\prime \prime}(0)=\frac{1}{2} A^{\prime \prime}(0),
$$

so that $B \in C^{1}(x \geqslant 0)$, as asserted.

Now set

$$
z(x)=w(x)-\bar{w}^{\prime}-\bar{w}
$$

then $z$ satisfies [cf. Eq. (1.4)] 


$$
r^{2} A z^{\prime \prime}+\Phi\left(\bar{w}^{\prime}+z^{\prime}\right)+w\left(1-w^{2}\right)=0
$$

If $u=1-w^{2}$, and $\bar{u}=1-\bar{w}^{2}$, then using Eq. (A2), we may write

$$
x z^{\prime \prime}+\frac{\Phi}{r^{2} B} z^{\prime}=\frac{\bar{u} \bar{w}-u w}{r^{2} B}-\frac{\Phi \bar{w}^{\prime}+\bar{u} \bar{w}}{r^{2} B} \equiv J,
$$

where, in view of Eq. (A3), $J \in C^{1}(x \geqslant 0)$. Thus

$$
x z^{\prime \prime}+z^{\prime}=\left(1-\frac{\Phi}{r^{2} B}\right) z^{\prime}+J
$$

Now as $\left(1-\left(\Phi / r^{2} B\right)\right) \in C_{0}^{1}(x \geqslant 0)$ and $z^{\prime} \in C_{0}^{0}(x \geqslant 0)$, we can write Eq. (A5) as

$$
\left(x z^{\prime}\right)^{\prime}-x \theta+J
$$

where $\theta \in C_{0}^{0}(x \geqslant 0)$. It follows from Lemma $\mathrm{A}_{1}$, that

$$
x z^{\prime}(x)=\int_{0}^{x} t \theta(t) d t+\int_{0}^{x} J(t) d t=x \xi(x)+\int_{0}^{x} J(t) d t
$$

where $\xi \in C_{0}^{1}(x \geqslant 0)$. Thus

$$
z^{\prime}(x)=\xi(x)+\frac{1}{x} \int_{0}^{x} J(t) d t
$$

and this shows that $z^{\prime} \in C_{0}^{1}(x \geqslant 0)$. Thus $w^{\prime} \in C^{1}(x \geqslant 0)$, so using Eqs. (1.3) and (4.9), we see that $A \in C_{0}^{3}(x \geqslant 0)$, and in Eq. (A6), $\theta \in C_{0}^{1}(x \geqslant 0)$ and $J \in C^{2}$. It follows that $z \in C^{3}$, and hence $(A, w) \in C^{3}(r \geqslant \rho) \times C^{3}(r \geqslant \rho)$. As was shown in Ref. 9, this means that $A$ and $w$ are analytic at $r=\rho$, so this solution coincides with a black-hole solution whose existence was proven in Ref. 9.1

${ }^{1}$ R. Bartnik and J. McKinnon, "Particle-like solutions of the Finstein-Yang-Mills equations," Phys. Rev. I ett. 61, $141-144$ (1988).

2 P. Bizon, "Colored black holes," Phys. Rev. Lett. 64, 2844-2847 (1990).

${ }^{3}$ P. Bizon and O. T. Popp. "No-hair theorem for spherical monopoles and dyons in SU(2) Einstein-Yang-Mills theory," Class. Quantum Gravit. 9, 193-205 (1992).

${ }^{4}$ P. Breitenlohner, P. Forgacs, and D. Maison, "Static spherically symmetric solutions of the Einstein-Yang-Mills equations," Commun. Math. Phys. 163, 141-172 (1994).

"A. A. Ershov and D. V. Galtsov, "Black holes in Einstein-Yang-Mills theory," Phys. Lett. A 150, 747 (1990).

${ }^{6}$ H. P. Kunzle and A. K. M. Masood-ul-Alam, "Spherically symmetric static SU(2) Einstein-Yang-Mills fields," J. Math. Phys. 31, 928-935 (1990).

7J. Smoller, A. Wasserman, S.-T. Yau, and B. McLeod, "Smooth static solutions of the Einstein/Yang-Mills equations," Commun. Math. Phys. 143, 115-147 (1991).

${ }^{8} \mathrm{~J}$. Smoller and A. Wasserman, "Existence of infinitely many smooth static, global solutions of the Einstein/Yang-Mills equations," Commun. Math. Phys. 151, 303-325 (1993).

9. Smoller, A. Wasserman, and S.-T. Yau, "Existence of black-hole solutions for the Einstein-Yang-Mills equations," Commun. Math. Phys. 154, 377-401 (1993).

${ }^{10} \mathrm{~J}$. Smoller and A. Wasserman, "An investigation of the limiting behavior of particlelike solutions to the Einstein-Yang/ Mills equations, and a new black-hole solution," Commun. Math. Phys. 161, 365-389 (1994).

"J. Smoller and A. Wasserman, "Limiting masses of solutions of Einstein-Yang-Mills equations" (to be published).

$12 \mathrm{~J}$. Smoller and A. Wasserman, "Uniqueness of extreme Reissner-Nördstrom solution in SU(2) Einstein-Yang-Mills Theory" (to be published)

${ }^{13}$ N. Straumann and Z. Zhou, "Instability of a colored black-hole solution," Phys. Lett. B 243, 33-35 (1990).

${ }^{14} \mathrm{R}$. M. Wald, "On the instability of the $n=1$ Einstein-Yang-Mills black holes, and mathematically related systems," J. Math. Phys. 33, 248-255 (1992).

${ }^{15}$ R. Adler, M. Bazin, and M. Schiffer, Introduction to General Relativity, 2nd ed. (McGraw Hill, New York, 1975).

${ }^{16}$ R. M. Wald, General Relativity (University of Chicago Press, Chicago, 1984). 